\title{
FACTORIZATIONS OF GENERIC MAPPINGS BETWEEN SURFACES
}

\author{
DOMENICO LUMINATI
}

(Communicated by Frederick R. Cohen)

\begin{abstract}
Given a generic mapping $F: \mathbf{S} \rightarrow \mathbf{N}$ of two smooth (i.e., $C^{\infty}$ ) real surfaces, $\mathbf{S}$ compact, and a line bundle $\pi: \mathbf{E} \rightarrow \mathbf{N}$, we look for necessary and sufficient conditions to find an immersion $\tilde{F}: \mathbf{S} \rightarrow \mathbf{E}$ such that $F=\pi \circ \widetilde{F}$.
\end{abstract}

\section{INTRODUCTION}

Let $\mathbf{S}, \mathbf{N}$ be two differentiable surfaces (i.e., real, $C^{\infty}, 2$-manifolds), $\mathbf{S}$ compact, and let $F: \mathbf{S} \rightarrow \mathbf{N}$ be a generic mapping, that is, a mapping locally equivalent to one of the following:

(i) $(x, y) \mapsto(x, y)$,

(ii) $(x, y) \mapsto\left(x, y^{2}\right)$,

(iii) $(x, y) \mapsto\left(x, y^{3}-x y\right)$,

and whose apparent contour is a smooth curve except for a finite number of normal crossings and semicubical cusps (compare $[6,1])$.

Let $\pi: \mathbf{E} \rightarrow \mathbf{N}$ be a differentiable line bundle (i.e., a rank 1 vector bundle). We shall answer the following:

Question. Does there exist an immersion (i.e., a mapping with injective differential at every point) $\widetilde{F}: \mathbf{S} \rightarrow \mathbf{E}$ such that $F=\pi \circ \widetilde{F}$ ? (i.e., the following is a commutative diagram):

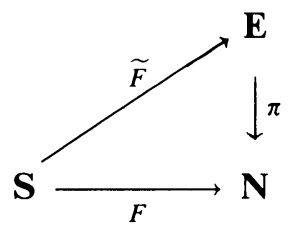

This question was first answered by Haefliger [2], in the case $\mathbf{N}=\mathbb{R}^{2}$ and $\mathbf{E}=\mathbb{R}^{3}$, and his theorem and proof were later generalized by Millet [3] to the case of an arbitrary surface $\mathbf{N}$ and $\mathbf{E}=\mathbf{N} \times \mathbb{R}$.

Received by the editors January 4, 1991.

1991 Mathematics Subject Classification. Primary 57R42, 57R45.

Key words and phrases. Surface, generic mapping, immersion, line bundle.

The author was partially supported by Ministero dell'Università e della Ricerca Scientifica e Technologica. 
In this paper we use again Haefliger's original idea to deal with the general case. In the first section, we state Theorem 1.1, which answers the question; in the second we give the proof of this theorem; and finally in the third section we apply it to the problem of finding a factorization of a generic mapping $F: \mathbf{S} \rightarrow \mathbb{R} \mathbb{P}^{2}$ by means of an immersion in $\mathbb{R} \mathbb{P}^{3}$ and a projection from a point.

\section{Statement of THE THEOREM}

Let $\Sigma$ denote the set of critical points of $F, C$ a connected component of $\Sigma$, and $f_{C}$ the restriction of $F$ to $C$. We can define the following two line bundles over the base space $C$ :

(1) $\kappa_{C}: K_{C} \rightarrow C$ the bundle of kernels of $d F$ (i.e., $\kappa_{C}^{-1}(p)=\operatorname{ker}(d F(p))$ $\forall p \in C)$

(2) $f_{C}^{*} \pi: f^{*} E \rightarrow C$ the induced bundle.

We shall prove the following.

Theorem 1.1. There exists an immersion $\widetilde{F}: \mathbf{S} \rightarrow \mathbf{E}$ such that $F=\pi \circ \widetilde{F}$ if and only if for all the components $C$ of $\Sigma$ the Whitney sum of the previous two bundles is trivial.

Remark. Let $\zeta: Z \rightarrow C$ be a line bundle over $C$, and define

$$
\varepsilon(\zeta)= \begin{cases}1 & \text { if } \zeta \text { is orientable } \\ -1 & \text { if } \zeta \text { is nonorientable }\end{cases}
$$

(In some sense this number is the Stiefel-Whitney class of the bundle.)

It is easily seen that the condition in Theorem 1.1 is equivalent to

$$
\varepsilon\left(\kappa_{C}\right) \varepsilon\left(f_{C}^{*} \pi\right)=1
$$

that is, either both bundles are orientable or both are nonorientable.

Furthermore, let $c(C)$ denote the number of cusp points in $C$, and $\nu_{C}$ : $N_{C} \rightarrow C$ the normal bundle of $C$ in $\mathbf{S}$. By [2, Lemma 1], we get

$$
\varepsilon\left(\kappa_{C}\right)=(-1)^{c(C)} \varepsilon\left(\nu_{C}\right)
$$

so condition (1.1) turns into

$$
(-1)^{c(C)} \varepsilon\left(\nu_{C}\right) \varepsilon\left(f_{C}^{*} \pi\right)=1
$$

Finally observe that, if $\pi: \mathbf{E} \rightarrow \mathbf{N}$ is the trivial bundle, then $\varepsilon\left(f_{C}^{*} \pi\right)=1$ and thus (1.2) reduces to Haefliger-Millet's condition.

\section{Proof of THE THEOREM}

First of all observe that finding a mapping $\widetilde{F}: \mathbf{S} \rightarrow \mathbf{E}$ such that $\pi \circ \widetilde{F}=F$ is, by the very definition of the induced bundle, the same as finding a cross-section $\sigma$ of the bundle $F^{*} \pi: F^{*} \mathbf{E} \rightarrow \mathbf{S}$, induced from $\pi: \mathbf{E} \rightarrow \mathbf{N}$ (see the diagram):

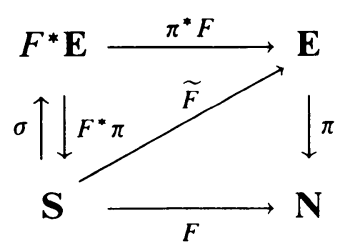


Now, if $\sigma$ is a section of $F^{*} \pi: F^{*} \mathbf{E} \rightarrow \mathbf{S}$, then

$$
\forall p \in \mathbf{S}-\Sigma d\left(\pi^{*} F \circ \sigma\right)(p) \text { is injective, }
$$

since a section is always an immersion and the set of critical points of $\pi^{*} F$ is $\left(F^{*} \pi\right)^{-1}(\Sigma)$. (Roughly speaking, the obstruction to making $\pi^{*} F \circ \sigma$ an immersion is "concentrated" around $\Sigma$.) This means that it suffices to find a section $\sigma_{1}$ of the bundle $\left.F^{*} \mathbf{E}\right|_{V}-V$ being a tubular neighborhood of $\Sigma$-such that $\pi^{*} F \circ \sigma_{1}$ is an immersion; an arbitrary extension of this section-which can always be found-will provide the desired immersion.

Since $V$ is a tubular neighborhood of $\Sigma$, it has the same number of connected components as $\Sigma$. Let $U$ be the connected component of $V$ containing $C$.

$U \cong \nu_{C}$ is diffeomorphic to the quotient

$$
\begin{array}{ll}
\mathbb{R}^{2} /(x, y) \sim(x+1, y) & \text { if } \varepsilon\left(\nu_{C}\right)=1, \\
\mathbb{R}^{2} /(x, y) \sim(x+1,-y) & \text { if } \varepsilon\left(\nu_{C}\right)=-1,
\end{array}
$$

in such a way that the curve $C$ is mapped onto the quotient of the line $\{y=0\}$.

Then the bundle $F^{*} \pi:\left.F^{*} \mathbf{E}\right|_{U} \rightarrow U$ is isomorphic to one of the following four:

(I) $\mathbb{R}^{3} /(x, y, z) \sim(x+1, y, z) \stackrel{\pi_{1}}{\rightarrow} \mathbb{R}^{2} /(x, y) \sim(x+1, y)$ if $\varepsilon\left(\nu_{C}\right)=1$ and $\varepsilon\left(f_{C}^{*} \pi\right)=1$;

(II) $\mathbb{R}^{3} /(x, y, z) \sim(x+1,-y, z) \stackrel{\pi_{2}}{\rightarrow} \mathbb{R}^{2} /(x, y) \sim(x+1,-y)$ if $\varepsilon\left(\nu_{C}\right)=-1$ and $\varepsilon\left(f_{C}^{*} \pi\right)=$ $1 ;$

(III) $\mathbb{R}^{3} /(x, y, z) \sim(x+1, y,-z) \stackrel{\pi_{3}}{\rightarrow} \mathbb{R}^{2} /(x, y) \sim(x+1, y)$ if $\varepsilon\left(\nu_{C}\right)=1$ and $\varepsilon\left(f_{C}^{*} \pi\right)=-1$;

(IV) $\mathbb{R}^{3} /(x, y, z) \sim(x+1,-y,-z) \stackrel{\pi_{4}}{\rightarrow} \mathbb{R}^{2} /(x, y) \sim(x+1,-y)$ if $\varepsilon\left(\nu_{C}\right)=-1$ and $\varepsilon\left(f_{C}^{*} \pi\right)=$ -1 ;

as follows from the fact that $U$ deforms onto $C$ and the Lifting Homotopy Theorem for fibre bundles (compare [5]), where each $\pi_{i}$ denotes the mapping induced by the canonical projection $(x, y, z) \mapsto(x, y)$.

Lemma 2.1. Every cross-section of the line bundle (I) [resp. (II), (III), (IV)] defines a function $h: \mathbb{R}^{2} \rightarrow \mathbb{R}$ such that (I) [resp. (II), (III), (IV)] holds:

(I) $\quad h(x+1, y)=h(x, y) \forall x, y$;

(II) $\quad h(x+1,-y)=h(x, y) \forall x, y$;

(III) $\quad h(x+1, y)=-h(x, y) \forall x, y$;

(IV) $\quad h(x+1,-y)=-h(x, y) \forall x, y$.

Conversely every such function defines a cross-section of the corresponding bundle.

Proof. Obvious.

Let $K_{x}$ denote the fiber of the bundle $\kappa: K \rightarrow C$ over $(x, 0)$ (i.e., the kernel of $d F(x, 0))$.

Lemma 2.2. Let $h$ be a function as in the previous lemma. Then $h$ defines $a$ cross-section $\sigma_{1}$ making $\pi^{*} F \circ \sigma_{1}$ an immersion if and only if

$$
\forall x \in \mathbb{R}, \forall(u, v) \in K_{x}-\{(0,0)\} \quad \frac{\partial h}{\partial x}(x, 0) u+\frac{\partial h}{\partial y}(x, 0) v \neq 0 .
$$

Proof. $\pi^{*} F \circ \sigma_{1}$ is an immersion if and only if

$$
\forall x \in \mathbb{R} \quad d \sigma_{1}(x, 0)\left[K_{x}\right] \nsubseteq \operatorname{ker}\left(d\left(\pi^{*} F\right)\left(\sigma_{1}(x, 0)\right)\right),
$$


but with our notation

$$
\begin{gathered}
d \sigma_{1}(x, 0)\left[K_{x}\right]=\left\{\left(u, v, \frac{\partial h}{\partial x}(x, 0) u+\frac{\partial h}{\partial y}(x, 0) v\right) \mid(u, v) \in K_{x}\right\} \\
\operatorname{ker}\left(d\left(\pi^{*} F\right)(x, 0, z)\right)=\left\{(u, v, w) \mid(u, v) \in K_{x}, w=0\right\}
\end{gathered}
$$

thus the thesis holds.

Lemma 2.3. Use the coordinates on $U \cong \nu_{C}$ given before Lemma 2.1. The line bundle $K$ is orientable (i.e., $\varepsilon\left(\kappa_{C}\right)=1$ ) if and only if there exists a never zero function $k: \mathbb{R} \rightarrow \mathbb{R}^{2}, \quad k(x)=\left(k_{1}(x), k_{2}(x)\right)$, such that

$$
\forall x \in \mathbb{R} \quad d F(x, 0)[k(x)]=0 ;
$$

$$
\forall x \in \mathbb{R} \quad k_{1}(x+1)=k_{1}(x) \quad \text { and } \quad k_{2}(x+1)= \begin{cases}k_{2}(x) & \text { if } \varepsilon\left(\nu_{C}\right)=1, \\ -k_{2}(x) & \text { if } \varepsilon\left(\nu_{C}\right)=-1 .\end{cases}
$$

On the contrary, $K$ is nonorientable (i.e., $\varepsilon\left(\kappa_{C}\right)=-1$ ) if and only if there exists a never zero function $k: \mathbb{R} \rightarrow \mathbb{R}^{2}, \quad k(x)=\left(k_{1}(x), k_{2}(x)\right)$, such that

$$
\forall x \in \mathbb{R} \quad d F(x, 0)[k(x)]=0 ;
$$

$$
\forall x \in \mathbb{R} \quad k_{1}(x+1)=-k_{1}(x) \quad \text { and } \quad k_{2}(x+1)= \begin{cases}-k_{2}(x) & \text { if } \varepsilon\left(\nu_{C}\right)=1, \\ k_{2}(x) & \text { if } \varepsilon\left(\nu_{C}\right)=-1\end{cases}
$$

Proof. A line bundle over $C$ is orientable if and only if it has a never zero crosssection, and such a section for the bundle $K$ is provided by a function $k$ as in (2.2). The second part of the statement is proved by a similar argument.

By Lemmas 2.1 and 2.2 and the considerations at the beginning of this section, it follows that proving Theorem 1.1 is the same as proving

Theorem 2.4. There exists a function $h$ satisfying condition (I) [resp. (II), (III), (IV)] of Lemma 2.1 and condition (2.1) of Lemma 2.2 if and only if (1.1) holds.

Proof $((1.1)$ is sufficient). There are four possibilities, corresponding to the four bundles (I), (II), (III), (IV).

(I), (II). Since $\varepsilon\left(f_{C}^{*} \pi\right)=1,(1.1)$ implies $\varepsilon(\kappa)=1$, so the assumption and thesis are the same as in [2, Lemma 2], thus the thesis holds.

(III). Since $\varepsilon\left(f_{C}^{*}\right)=-1,(1.1)$ implies $\varepsilon(\kappa)=-1$. Then by (2.3) we have a never zero function $k$ such that

$$
\forall x \in \mathbb{R} \quad k_{1}(x+1)=-k_{1}(x) \quad \text { and } \quad k_{2}(x+1)=-k_{2}(x) .
$$

Define

$$
h(x, y)=r(x)+y k_{2}(x)
$$

where

$$
r(x)=\int_{0}^{x} k_{1}(t) d t-\frac{1}{2} \int_{0}^{1} k_{1}(t) d t
$$


Observe that

$$
\begin{aligned}
r(x+1)+r(x) & =\int_{0}^{x+1} k_{1}(t) d t+\int_{0}^{x} k_{1}(t) d t-\int_{0}^{1} k_{1}(t) d t \\
& =\int_{0}^{x} k_{1}(t) d t+\int_{1}^{x+1} k_{1}(t) d t \\
& =\int_{0}^{x} k_{1}(t) d t+\int_{0}^{x} k_{1}(t+1) d t \quad \text { [by (2.4)] } \\
& =\int_{0}^{x} k_{1}(t) d t-\int_{0}^{x} k_{1}(t) d t=0 ;
\end{aligned}
$$

and using (2.4) again we have

$$
h(x+1, y)=-h(x, y) \quad \forall x, y,
$$

that is, condition (III) holds. Furthermore $\nabla h(x, 0)=k(x)$, so $(2.1)$ holds too.

(IV). Once again (1.1) implies $\varepsilon\left(\kappa_{C}\right)=-1$; therefore, by (2.3) we have a never zero function $k$ such that

$$
\forall x \in \mathbb{R} \quad k_{1}(x+1)=-k_{1}(x) \quad \text { and } \quad k_{2}(x+1)=k_{2}(x) .
$$

As before define $h(x, y)=r(x)+y k_{2}(x)$, where

$$
r(x)=\int_{0}^{x} k_{1}(t) d t-\frac{1}{2} \int_{0}^{1} k_{1}(t) d t,
$$

and use (2.5) to get

$$
h(x+1,-y)=-h(x, y), \quad \nabla h(x, 0)=k(x),
$$

that is, the thesis.

((1.1) is necessary). Suppose that such a function $h$ exists. Then the projection of $\nabla h(x, 0)$ on $K_{x}$ will provide a never zero function $k$ such that either (2.2) -in cases (I) or (II) - or (2.3) - in cases (III) or (IV)-holds. This ends the proof of the theorem.

\section{GeNERIC MAPPINGS IN $\mathbb{R P}^{2}$}

Let $\mathbb{R} \mathbb{P}^{3}$ denote projective space, and let $p \in \mathbb{R} \mathbb{P}^{3}$ be a fixed point. Identify $\mathbb{R} \mathbb{P}^{2}$ with the set of lines in $\mathbb{R P}^{3}$ through the point $p$. There is a canonical projection

$$
\pi: \mathbb{R} \mathbb{P}^{3}-\{p\} \rightarrow \mathbb{R} \mathbb{P}^{2}
$$

Let $F: \mathbf{S} \rightarrow \mathbb{R} \mathbb{P}^{2}$ be a generic mapping. One can ask for the existence of an immersion $\widetilde{F}: \mathbf{S} \rightarrow \mathbb{R P}^{3}-\{p\}$ such that $F=\pi \circ \widetilde{F}$.

Proposition 3.1. With the just said assumptions and notation, such an $\widetilde{F}$ exists if and only if for all connected components $C$ of the critical set $\Sigma$ of $F$

$$
(-1)^{c(C)} \varepsilon\left(\nu_{C}\right)=1 \text {. }
$$

Remark. The condition is exactly the same found by Haefliger [2] looking for a factorization with an immersion in $\mathbb{R}^{3}$ of a generic mapping in $\mathbb{R}^{2}$. 


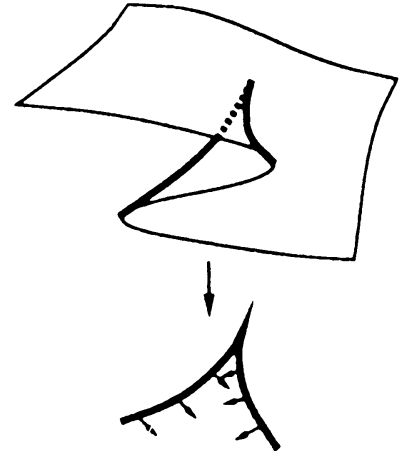

FIGURE 1

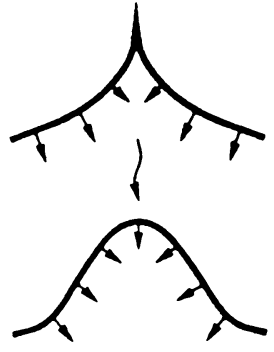

Figure 2

Proof. Observe that the projection $\pi: \mathbb{R P}^{3}-\{p\} \rightarrow \mathbb{R} \mathbb{P}^{2}$ is nothing but the tautological bundle over $\mathbb{R} \mathbb{P}^{2}$; thus we are allowed to use Theorem 1.1 and the thesis will be proved once we show that $f_{C}^{*} \pi: f_{C}^{*} \mathbf{E} \rightarrow C$ is the trivial bundle for all $C$. But this is a consequence of the following:

Lemma 3.2. For all components $C$ of $\Sigma$ the curve $f_{C}: C \rightarrow \mathbb{R} \mathbb{P}^{2}$ is homotopically trivial.

Proof. First of all, observe that the curve $f_{C}$ is sided, which means it possesses a field of transverse vectors, excepted at cusp points, that in a neighborhood of each cusp is directed towards the internal part of the cusp. Such a field can be defined by the direction toward which the map $F$ folds (see Figure 1).

It is not hard to see that the curve $f_{C}$ can be deformed, by means of an homotopy, to a regular (i.e., with never zero derivative) sided curve (see Figure 2). Thus, to prove the lemma, it is enough to prove the following:

Lemma 3.3. Any regular sided closed curve in the projective plane is homotopically trivial.

Proof. Let $f:[0,1] \rightarrow \mathbb{R} \mathbb{P}^{2}$ be such a curve, that is,

$$
f(1)=f(0), \quad f^{\prime}(1)=f^{\prime}(0),
$$

and let $n(t)$ be a field of transverse vectors along $f$.

Let $\tilde{f}$ be the lifting of $f$ to the sphere. Then it is easily seen that $f$ is homotopically trivial if and only if $\tilde{f}$ is a closed curve. Suppose, for the sake of contradiction, $\tilde{f}(0) \neq \tilde{f}(1)$, and let $\tilde{n}(t)$ denote the lifting of the vector field $n$.

Since the quotient map from the sphere to the projective plane identifies opposite points and opposite tangent vectors at opposite points, we have that the following hold:

$$
\tilde{f}(1)=-\tilde{f}(0), \quad \tilde{f}^{\prime}(1)=-\tilde{f}^{\prime}(0), \quad \tilde{n}(1)=-\tilde{n}(0) .
$$

By our assumptions, the three vectors $\tilde{f}(t), \tilde{f}^{\prime}(t)$, and $\tilde{n}(t)$ are linearly independent for all $t \in[0,1]$; therefore,

$$
\operatorname{det}\left(\tilde{f}(t)\left|\tilde{f}^{\prime}(t)\right| \tilde{n}(t)\right) \neq 0 \quad \forall t \in[0,1]
$$


on the other hand, using (3.1) we have

$$
\operatorname{det}\left(\tilde{f}(1)\left|\tilde{f}^{\prime}(1)\right| \tilde{n}(1)\right)=-\operatorname{det}\left(\tilde{f}(0)\left|\tilde{f}^{\prime}(0)\right| \tilde{n}(0)\right),
$$

and this is a contradiction.

This ends the proof of the two lemmas and of Proposition 3.1.

Remark. The converse of Lemma 3.3 is also true. In fact if $f$ is homotopically trivial, then it has a closed lifting $\tilde{f}$ to the sphere. Since the sphere is orientable, $\tilde{f}$ possesses a field $\tilde{n}$ of transverse vectors. The covering map transforms $\tilde{n}$ to a field of transverse vectors along $f$ (see also [4, Proposition 1]).

\section{ACKNOWLEDGMENT}

I wish to thank Professor Riccardo Benedetti, of the University of Pisa, for the fruitful discussions and suggestions that aroused my interest in this matter. I also wish to thank the referee, whose remarks have been of great help in smoothing the style and improving the readability of this paper.

\section{REFERENCES}

1. T. Bröcker and L. Lander, Differentiable germs and catastrophes, London Math. Soc. Lecture Note Ser., vol. 17, Cambridge Univ. Press, Cambridge, 1975.

2. A. Haefliger, Quelques remarques sur les applications differentiables d'une surface dans le plan, Ann. Inst. Fourier (Grenoble) 10 (1960), 47-60.

3. K. C. Millet, Generic smooth maps of surfaces, Topology Appl. 18 (1984), 197-215.

4. R. Pignoni, On surfaces and their contours, Manuscripta Math. 72 (1991), 223-249.

5. N. Steenrod, The topology of fibre bundles, Princeton Univ. Press, Princeton, NJ, 1951.

6. H. Whitney, On singularities of mappings of Euclidean space. I, Ann. of Math. (2) 62 (1955), 374-410. ITALY

E-mail address: luminati@dm.unipi.it 